\title{
Asking and Answering Questions to Evaluate the Factual Consistency of Summaries
}

\author{
Alex Wang* \\ New York University \\ a lexwang@nyu.edu
}

\author{
Kyunghyun Cho \\ Facebook AI \\ New York University \\ CIFAR Associate Fellow \\ kyunghyun. cho@nyu.edu
}

\author{
Mike Lewis \\ Facebook AI \\ mikelewis@fb.com
}

\begin{abstract}
Practical applications of abstractive summarization models are limited by frequent factual inconsistencies with respect to their input. Existing automatic evaluation metrics for summarization are largely insensitive to such errors. We propose QAGS, ${ }^{1}$ an automatic evaluation protocol that is designed to identify factual inconsistencies in a generated summary. QAGS is based on the intuition that if we ask questions about a summary and its source, we will receive similar answers if the summary is factually consistent with the source. To evaluate QAGS, we collect human judgments of factual consistency on model-generated summaries for the CNN/DailyMail (Hermann et al., 2015) and XSUM (Narayan et al., 2018) summarization datasets. QAGS has substantially higher correlations with these judgments than other automatic evaluation metrics. Also, QAGS offers a natural form of interpretability: The answers and questions generated while computing QAGS indicate which tokens of a summary are inconsistent and why. We believe QAGS is a promising tool in automatically generating usable and factually consistent text. Code for QAGS will be available at https://github. $\mathrm{com} /$ W4ngatang/qags.
\end{abstract}

\section{Introduction}

Automatic summarization aims to produce summaries that are succinct, coherent, relevant, and crucially - factually correct. Recent progress in conditional text generation has led to models that can generate fluent, topical summaries (Lewis et al., 2019). However, model-generated summaries frequently contain factual inconsistencies, limiting their applicability (Kryscinski et al., 2019a).

The problem of factual inconsistency is due in part to the lack of automatic evaluation metrics that can detect such errors. Standard metrics for

\footnotetext{
${ }^{1}$ Pronounced "kags".
}

evaluating generated text are predominantly based on counting $n$-grams, which weigh all $n$-grams equally and are insensitive to semantic errors. This inadequacy leaves human evaluation as the primary method for evaluating the factual consistencies, which has been noted to be challenging even for humans (Daume III and Marcu, 2005; Kryscinski et al., 2019b), in addition to being slow and costly.

We argue that evaluation metrics that are able to capture subtle semantic errors are required to build better models. In this work, we introduce a general framework for evaluating conditional text generation that is designed to detect factual inconsistencies in generated text with respect to some input. Our framework consists of three steps: (1) Given a generated text, a question generation (QG) model generates a set of questions about the text. (2) We then use question answering (QA) models to answer these questions given both the input and the generated text. (3) A quality score is computed based on the similarity of corresponding answers.

This approach leverages recent progress in QA and QG to ask and answer human readable, ontopic questions (Devlin et al., 2019; Song et al., 2019). It only assumes access to a question answering dataset to train the QG and QA models, and is applicable to any modality where a QA model is available, e.g. text, images, or knowledge graphs.

We use this framework to develop QAGS (Question Answering and Generation for Summarization), a metric for evaluating the factual consistency of abstractive document summaries. Compared to commonly used automatic metrics such as ROUGE (Lin, 2004), QAGS shows dramatically higher correlations with human judgements of factuality, for example achieving a Pearson correlation coefficient of 54.52 on the CNN/DailyMail summarization task, compared to 17.72 for ROUGE-2. QAGS also achieves new state-of-the-art results on evaluating the factuality of summaries, outper- 
forming recently proposed NLI models for this task (Kryscinski et al., 2019b).

Finally, we analyse the robustness of QAGS through an ablation study. QAGS shows robustness to the quality of the underlying QG and QA models, the domain of the models, and the number of questions asked. Even under the worst ablation settings, QAGS still has stronger correlation with human judgments than other automatic metrics.

Overall, we contribute the following: (1) We introduce QAGS, an automatic model-based evaluation metric for measuring the factual consistency of model-generated text. (2) We collect a new set of human judgments of factual consistency of model-generated summaries for two summarization datasets. We demonstrate that QAGS correlates with these judgments significantly better than other automatic metrics. (3) We show via ablations that QAGS is robust to a number of factors including underlying model quality and domain mismatch. (4) We analyze the questions and answers produced in computing QAGS to illustrate which parts of summaries are inconsistent. (5) We will release models and code to compute QAGS.

\section{Background: Automatically Evaluating Machine Generated Text}

Standard approaches to evaluating generated text are primarily based on counting $n$-gram overlap. These methods assume access to one or more reference texts, and score a generated summary based on the precision and recall of all reference $n$-grams in the generated summary. We briefly describe the most common metrics in this family, and refer readers to Liu et al. (2016) for further discussion.

ROUGE (Lin, 2004) was developed specifically for evaluating automatic summarization, and its variants are the de facto standard for such. The most common variant is ROUGE- $n$ (typically $n \in$ $\{1,2\}$ ), which computes the F1 score for all reference $n$-grams in the generated summary. ROUGE$L$, another commonly used variant, is the length of the longest common subsequence (possibly nonconsecutive) between a summary and references.

BLEU (Papineni et al., 2002) is closely related to ROUGE but was developed for machine translation. BLEU computes the precision of the reference $n$ grams in the generated summary. METEOR (Lavie and Agarwal, 2007) extends BLEU by using an alignment between the generated text and a reference, as well as using stemming and synonym replacement for more flexible $n$-gram matching.

We identify two key deficiencies when using these $n$-gram based evaluation metrics to detect factual inconsistencies in generated text.

First, these metrics require one or more reference texts to compare against. Obtaining references can be expensive and challenging, and as such many text generation datasets contain only a single reference. This problem is exacerbated with highentropy generation tasks, such as summarization or dialogue, where there is a very large number of acceptable outputs. In these settings, comparing against a single reference is woefully inadequate.

Second, given a reference to compare against, $n$-gram based approach weigh all portions of the text equally, even when only a small fraction of the $n$-grams carry most of the semantic content. Factual inconsistencies caused by minor changes may be drowned out by otherwise high $n$-gram overlap, making these metrics insensitive to these errors. For example, the sentences "I am writing my paper in Vancouver." and "I am not writing my paper in Vancouver." share nearly all unigrams and bigrams despite having the opposite meaning.

\section{A Framework for Automatically Evaluating Factual Consistency}

We introduce a framework for automatically detecting factual inconsistencies in generated text while also addressing the deficiencies of current approaches. Let $X$ and $Y$ be sequences of tokens coming from a vocabulary $V$ where $X$ is a source text and $Y$ is a summary of $X$. We define $p(Q \mid Y)$ as a distribution over all possible questions $Q$ given summary $Y$, and $p(A \mid Q, X)$ and $p(A \mid Q, Y)$ as distributions over all possible answers $A$ to a particular question $Q$ given either the source $X$ or the summary $Y$. We constrain the questions $Q$ and answers $A$ to also be sequences of tokens from $V$. Then the factual consistency of the summary $Y$ is

$$
E_{Q \sim p(Q \mid Y)}[D(p(A \mid Q, X), p(A \mid Q, Y))],
$$

where $D$ is some function measuring the similarity of the two answer distributions. This expression is maximized when $Y$ contains a subset of the information in $X$ such that it produces the same answer for any question from $p(Q \mid Y)$. This happens trivially when $Y=X$, i.e. we take $X$ as its own summary, but in many cases this solution is unacceptable. 


\section{Source}

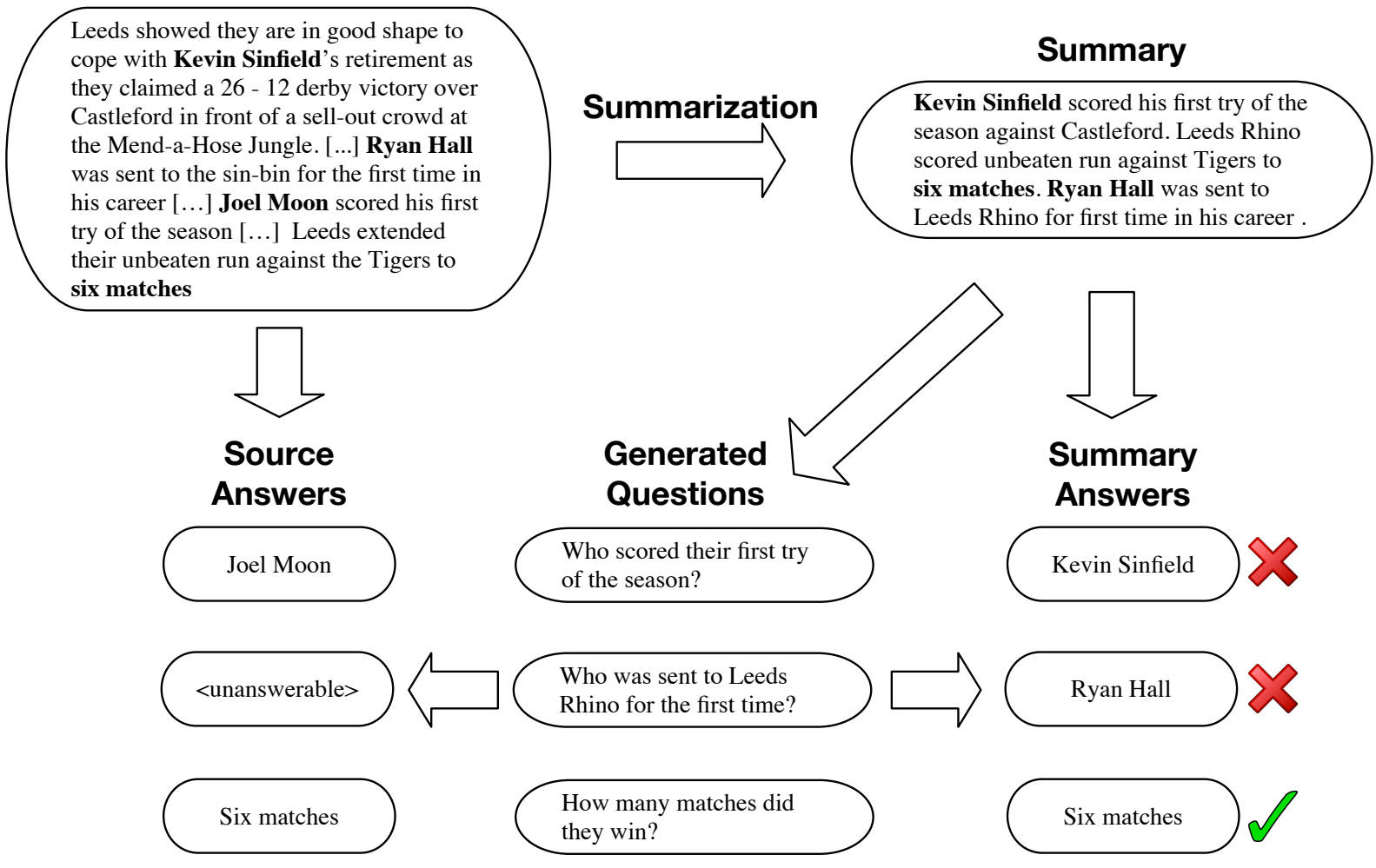

Figure 1: Overview of QAGS. A set of questions is generated based on the summary. The questions are then answered using both the source article and the summary. Corresponding answers are compared using a similarity function and averaged across questions to produce the final QAGS score.

This framework addresses the two issues with $n$ gram based approaches. Instead of requiring a reference to compare against, our framework asks questions based on the generation itself, and compares answers with the provided source text. Also, the use of questions focuses the metric on the semantically relevant parts of the generated text, rather than weighting all parts of the text equally.

In practice, exactly computing the expectation in Equation 1 is intractable due to the large space of possible questions. One potential workaround is to randomly sample questions from $p(Q \mid Y)$, but this suffers from high variance and requires many samples to obtain a good estimate. Instead, we focus on producing highly probable questions, e.g. as produced by beam search, which may be biased in the limit, but will require fewer questions to estimate because of the higher quality of the questions.

\section{QAGS}

Using this framework requires specifying the question distribution $p(Q \mid Y)$, the answer distributions $p(A \mid Q, *)$, and the answer similarity function $D$. We apply this framework to summarization to de- velop QAGS and describe our instantiations of these components.

Question Generation To instantiate $p(Q \mid Y)$, we draw on recent work on automatic question generation $(\mathrm{QG})$, which models this distribution using neural seq2seq models (Du et al., 2017; Krishna and Iyyer, 2019). We over-sample questions, and then filter out low quality questions as follows.

First, we train and generate from answerconditional QG models. During training, the model receives both the answer and the source article, and is trained to maximize the likelihood of the paired question. At test time, given a summary $Y$, we determine candidate answers. We condition on these answers and the summary to generate questions.

Next, we filter out low-quality questions using a number of heuristics, such as duplicates and questions less than three tokens long. We also found it especially useful to run the QA model (see next section) on all of the candidate questions, and filter out questions for which the QA model predicted no answer or a different answer than expected. 
Question Answering We instantiate the answer distributions $p(A \mid Q, *)$ as extractive QA models, for simplicity. In using extractive QA models, we assume the facts are represented as text spans in the article and summary. Future work should explore using abstractive QA models, which could match paraphrases of the same answer.

Answer Similarity We use token-level F1 to compare answers, which is standard for extractive QA and equivalent to defining $D$ as

$$
F 1(\arg \max p(A \mid Q, X), \arg \max p(A \mid Q, Y))
$$

The QAGS Score Given these components, we obtain the QAGS score of a generation by (1) generating $K$ questions conditioned on the summary, (2) answering the questions using both the source article and the summary to get two sets of answers, (3) comparing corresponding answers using the answer similarity metric, and (4) averaging the answer similarity metric over all questions. We depict this process in Figure 1.

\section{Experiments}

\subsection{Human Evaluation}

We test whether QAGS accurately measures the factual consistency of a summary with respect to a source article by computing correlations with human judgments of factual consistency.

Datasets We focus on abstractive summarization, which is particularly interesting because factual consistency with the original text is crucial to usability, and a lack of such consistency has plagued abstractive neural summarization models (Cao et al., 2018; Falke et al., 2019; Kryscinski et al., 2019b, i.a.). To compare with prior work on evaluating summarization, we use two common abstractive summarization datasets, CNN/Daily Mail (CNNDM, Hermann et al., 2015; Nallapati et al., 2016) and XSUM (Narayan et al., 2018).

CNN/DM is a standard dataset for summarization that consists of CNN and DailyMail articles. Each reference summary consists of the concatenation of three editor-written, bullet point highlights. For summaries, we use 235 test outputs from Gehrmann et al. (2018).

XSUM was created by taking the first sentence of a news article as the summary, and using the rest of the article as the source. Consequently, XSUM summaries are significantly more abstractive than

\begin{tabular}{lcc}
\hline Metric & CNN/DM & XSUM \\
\hline ROUGE-1 & 28.74 & 13.22 \\
ROUGE-2 & 17.72 & 8.95 \\
ROUGE-L & 24.09 & 8.86 \\
METEOR & 26.65 & 10.03 \\
BLEU-1 & 29.68 & 11.76 \\
BLEU-2 & 25.65 & 11.68 \\
BLEU-3 & 23.96 & 8.41 \\
BLEU-4 & 21.45 & 5.64 \\
BERTScore & 27.63 & 2.51 \\
QAGS & $\mathbf{5 4 . 5 3}$ & $\mathbf{1 7 . 4 9}$ \\
\hline
\end{tabular}

Table 1: Summary-level Pearson correlation coefficients between various automatic metrics and human judgments of correctness for summarization datasets. All correlations are significant at $p<.01$ and $p<.05$ for CNN/DM and XSUM, respectively. QAGS obtains substantially higher correlations than all other automatic metrics.

those of CNN/DM, and extractive summarization models perform poorly on this dataset.

We found that while the XSUM summaries are more abstractive, frequently there are facts (e.g. first names) in the summary that are not available in the "article". This quirk made it especially difficult for humans and QAGS to tell when factual errors were being made by the summarization model. To remedy this, for human evaluation and QAGS, we prepend the summary back to the "article". We use a subset of 239 test outputs from BART fine-tuned on XSUM (Lewis et al., 2019).

Annotation Protocol We collect human judgments on Amazon Mechanical Turk ${ }^{2}$ via ParlAI (Miller et al., 2017). We present summaries one sentence at a time, along with the entire article. For each summary sentence, the annotator makes a binary decision as to whether the sentence is factually consistent with the article. Workers are instructed to mark non-grammatical sentences as not consistent, and copies of article sentences as consistent. Workers are paid $\$ 1$ per full summary annotated. See Appendix A for further details.

We collect 3 annotations per summary. To obtain a single consistency score per summary, we first take the majority vote for each sentence, then average the binary scores across summary sentences to produce a final score.

Inter-annotator agreement as measured by Krip-

\footnotetext{
${ }^{2}$ https://www.mturk.com/
} 
pendorff's $\alpha$ is 0.51 and 0.34 for CNN/DM and XSUM, respectively indicating "moderate" and "fair" agreement (Ageeva et al., 2015). While not perfect, these agreement numbers are in-line with similar figures from previous work on summarization evaluation (Daume III and Marcu, 2005).

\subsection{Experimental Details}

Question Generation We train answerconditional QG models by fine-tuning a pretrained BART language model (Lewis et al., 2019) on NewsQA (Trischler et al., 2017), a dataset consisting of $\mathrm{CNN}$ articles and crowdsourced questions. During training, the model receives the concatenation of the source article and an answer, and is trained to predict the question. The answer, source article, and question are concatenated with intervening special tokens to mark the boundaries.

At test time, the model receives the concatentation of a summary and an expected answer, and outputs question candidates. For each summary, we extract 10 named entities and noun phrases as answer candidates using the en-web-sm spaCy model.$^{3}$ For each summary-answer pair, we generate questions using beam search with width 10 , for a total of 100 question candidates. We experimented with generating via top- $k$ (Holtzman et al., 2019) and top- $p$ (Fan et al., 2018) sampling, but the generated questions, while diverse, were noisy and frequently nongrammatical. After filtering, we use the $K=20$ most probable questions. If a summary has too few filtered questions, we randomly sample questions to reach the required number. For additional filtering and training details, see Appendix B. We implement these models with fairseq (Ott et al., 2019).

Question Answering We train extractive QA models by fine-tuning BERT (Devlin et al., 2019) on SQuAD2.0 (Rajpurkar et al., 2018). We use the large-uncased BERT variant via the transformers library (Wolf et al., 2019).

We found that allowing the model to predict that a question is unanswerable, as is the case in SQuAD2.0, is particularly useful in filtering out bad questions, as questions based on hallucinated facts in the summary should be unanswerable using the source article.

Baselines We compare against a number of automatic evaluation metrics: ROUGE (Lin, 2004),

\footnotetext{
${ }^{3}$ https://spacy.io/api/entityrecognizer
}

METEOR (Lavie and Agarwal, 2007), BLEU (Papineni et al., 2002), and BERTScore (Zhang et al., 2019). The latter uses BERT representations to compute an alignment between generation and reference tokens, and which is then used to compute a soft version of unigram F1. We use the large-uncased BERT variant.

\subsection{Results}

We present Pearson correlations between humanjudged consistency scores and various automatic metrics in Table 1. For CNN/DM, all results are significant with $p<0.01$; for XSUM, all results are significant with $p<.05$. QAGS strongly outperforms other automatic evaluation metrics in terms of correlation with the summary-level human judgments of factual consistency. BLEU and ROUGE perform comparably, and lower order $n$-gram metrics work better. BERTScore matches the best $n$ gram metrics on CNN/DM, but the worst overall on XSUM.

On CNN/DM, QAGS obtains nearly twice the correlation of the next best automatic metric (BLEU-1). We speculate that this large increase is due to the sensitivity of the QA model to the sentence fusing behavior exhibited in many summarization models trained on CNN/DM (Lebanoff et al., 2019). When two sentences are fused to produce an incorrect summary statement, the QA model produces different answers when using the source article than when using the summary.

On XSUM, all metrics correlate worse with human judgments than on CNN/DM, which reflects the fact that XSUM is more abstractive. QAGS still outperforms the next best automatic metric.

\subsection{Ablations}

A potential issue with model-based evaluation is that the quality of the evaluation metric may depend heavily on specific hyperparameter settings. We explore the extent to which this is true with QAGS by performing ablations on several factors.

Model Quality We first consider the degree to which the quality of the underlying models impacts their evaluation capabilities.

For QA quality, we answer this question by training QA models of varying quality by finetuning different versions of BERT on SQuAD. We present results in Table 2. The QA models perform similarly despite substantially different performances on the SQuAD develop- 


\begin{tabular}{lccc}
\hline QA model & $\begin{array}{c}\text { SQuAD } \\
(\text { F1 })\end{array}$ & $\begin{array}{c}\text { CNN/DM } \\
\text { (Pear.) }\end{array}$ & $\begin{array}{c}\text { XSUM } \\
\text { (Pear.) }\end{array}$ \\
\hline bert-base & 75.95 & 55.20 & 20.71 \\
bert-large & 81.57 & 54.53 & 17.49 \\
bert-large-wwm & 84.36 & 51.36 & 18.07 \\
\hline
\end{tabular}

Table 2: Pearson correlations between human judgments of factual consistency and QAGS using QA models of different qualities, as measured by performance on the SQuAD2.0 development set (F1). The correlations are stable across QA model quality.

\begin{tabular}{ccc}
\hline $\begin{array}{c}\text { NewsQA } \\
\text { (ppl.) }\end{array}$ & $\begin{array}{c}\text { CNN/DM } \\
\text { (Pear.) }\end{array}$ & $\begin{array}{c}\text { XSUM } \\
\text { (Pear.) }\end{array}$ \\
\hline 5.48 & 54.53 & 17.49 \\
9.50 & 50.09 & 19.93 \\
18.56 & 47.92 & 16.38 \\
\hline
\end{tabular}

Table 3: Pearson correlations between human judgments of factual consistency and QAGS with QG models of varying quality, as measured by perplexity on the NewsQA development set. We see some decrease in correlation on CNN/DM as QG perplexity increases, though we do not see a similar trend for XSUM.

ment set. Surprisingly, using the best QA model (bert-large-wwm) does not lead to the best correlations with human judgments. On CNN/DM, bert-large-wwm slightly underperforms bert-base and bert-large. On XSUM, bert-base slightly outperforms the other two BERT variants. These results indicate that QAGS is fairly robust to the quality of the underlying QA model, though we note that BERT is a strong QA baseline, and using weaker QA models might lead to larger performance dropoffs.

To ablate QG quality, we use models with increasing perplexity on the NewsQA development set. Results in Table 3 show that QAGS is robust to the QG model quality, with some decrease in correlation with human judgments as perplexity increases on CNN/DM, and no clear trend on XSUM. Even the weakest QG model still significantly outperforms all other automatic metrics in Table 1.

Domain Effects Our approach relies on having a labeled dataset to train QG and QA models. However, for relatively niche domains, such a labeled QA/QG dataset may not exist. Instead, we may need to resort to using models trained on outof-domain data, leading to domain shift effects that negatively impact the quality of the QAGS scores. We simulate this setting by fine-tuning the

\begin{tabular}{ccc}
\hline \# Questions & CNN/DM & XSUM \\
\hline 5 & 41.61 & 15.63 \\
10 & 41.17 & 15.49 \\
20 & 54.53 & 17.49 \\
50 & 57.94 & 17.74 \\
\hline
\end{tabular}

Table 4: Pearson correlation coefficients between QAGS scores with varying number of questions and human judgments of correctness for summarization datasets. The correlation increases with the number of questions used, but with decreasing marginal benefit.

QG model on SQuAD, which is of similar size to NewsQA but drawn from Wikipedia articles rather than CNN articles, which exactly matches the genre of the summarization datasets.

Evaluating with this QG model, we get correlations of 51.53 and 15.28 with human judgments on CNN/DM and XSUM respectively, versus 54.53 and 17.49 when using the NewsQA-tuned QG model. The drop in performance indicates a negative domain shift effect. However using the SQuAD-tuned QG model still substantially outperforms all other automatic metrics, again pointing to the robustness of QAGS.

Number of Questions Next, we investigate the correlation with human judgments when varying the number of questions used. Results in Table 4 show that increasing the number of questions used improves correlations with human judgments. We observe a large increase when moving from 10 to 20 questions, and a smaller increase from 20 to 50 questions, indicating decreasing marginal benefit moving beyond 50 questions. However, we observe frequent clusters of generated questions that only differ by a few tokens. Encouraging greater diversity when generating questions might lead to better correlations when more questions are used. Still, With just 5 questions used QAGS substantially outperforms other automatic metrics, which indicates its robustness.

Answer Similarity Metric Finally, we consider using exact match as an alternative answer similarity metric. Exact match is another common evaluation metric for extractive QA, and is more restrictive than F1. When using EM, we obtain Pearson correlations with human judgments of 45.97 and 18.10 on CNN/DM and XSUM, as opposed to 54.53 and 17.49 when using F1. 


\begin{tabular}{lc}
\hline Model/Metric & \% Correct $(\uparrow)$ \\
\hline Random & $50.0 \%$ \\
BERT NLI & $64.1 \%$ \\
ESIM & $67.6 \%$ \\
FactCC & $70.0 \%$ \\
QAGS & $\mathbf{7 2 . 1 \%}$ \\
\hline
\end{tabular}

Table 5: Results on the sentence ranking task from Falke et al. (2019). Results using BERT NLI and ESIM are from Falke et al. (2019); FactCC is from Kryscinski et al. (2019b). QAGS outperforms previous work.

\section{Re-ranking with QAGS}

Several works explore the use of natural language inference (NLI) models to detect factual consistency in generated text (Welleck et al., 2019; Falke et al., 2019). We compare against these methods by evaluating on the sentence ranking experiment from Falke et al. (2019). The experiment uses 373 triplets of source sentences from CNN/DM and two summary sentences generated from the model from Chen and Bansal (2018). One summary sentence is factually consistent with the source sentence, and the other is inconsistent. A metric (or model) is evaluated based on how often it ranks the consistent sentence higher than the inconsistent sentence.

We present the results in Table 5. Results using two NLI models fine-tuned on MultiNLI (Williams et al., 2018), BERT NLI, and ESIM (Chen et al., 2017), are from Falke et al. (2019). FactCC (Kryscinski et al., 2019b) is an NLI-based factchecking model that is trained on a dataset tailor made for detecting factual inconsistencies in generated text. QAGS outperforms these methods, while requiring no special supervision for this task.

\section{Qualitative Analysis}

Interpreting QAGS The questions and answers produced in computing QAGS are directly interpretable, and highlight errors in summaries. We present examples of articles, summaries, and the QAGS questions and answers in Table 6.

On the first example (Table 6, top), QAGS detects several factual inconsistencies in the generated summary: The summary mistakes the first name of the attacker, the location of the attack, and the weapons used. Because the QG model focuses on these details, QAGS is able to correctly penalize the summary for its hallucinations. Because the answer candidates used are mostly named entities and noun phrases, QAGS is particularly effective at detecting errors of this kind. Using more diverse answer candidates may broaden the set of inconsistencies that QAGS is able to detect.

The second example (Table 6, bottom), illustrates failure modes of QAGS. For example, the QA model incorrectly marks question 2 as unanswerable. On question 4, both answers produced are correct, but because they have no common tokens, they are marked inconsistent by QAGS.

Error Analysis The interpretability of QAGS allows for error analysis on the metric. We manually annotate 400 triplets of generated questions, article answers, and summary answers that are produced in computing QAGS on the XSUM summaries, and label them by the quality of the generated questions, predicted answers, and answer similarity scores.

Among the generated questions, $8.75 \%$ are nonsensical, while $3.00 \%$ are well-formed but unanswerable using the generated summary they were conditioned upon. These figures indicate that the vast majority of questions are understandable and on-topic. We frequently observe multiple questions with slightly different wordings, which is likely due to the low number of answer candidates in XSUM summaries (which are one sentence long) and due to beam search. $8.25 \%$ of questions are well-formed but unanswerable using the source, which is usually due to a hallucinated fact in the summary that the QG model turns into a question.

Among predicted answers, $1.75 \%$ of questions are potentially answerable using the summary, but are incorrectly answered. This percentage increases to $32.50 \%$ for the article, which indicates that the transfer ability of the QA model is lacking. In a small number of cases, we found that while a question had a single answer in the summary, it could have multiple answers in the article.

Finally, for $8.00 \%$ of the examples, the question is answered correctly using both the article and summary, but the answers have high lexical variation such that $\mathrm{F} 1$ score fails to detect their similarity. While this happens in a relatively small number of cases, exploring similarity metrics other than $n$-gram based approaches could be useful.

Limitations We emphasize that QAGS and our overall framework are specifically designed to detect factual inconsistencies in generated summaries relative to the source article. QAGS does not measure other desirable properties of generated text, 
Article: On Friday, 28-year-old Usman Khan stabbed reportedly several people at Fishmongers' Hall in London with a large knife, then fled up London Bridge. Members of the public confronted him; one man sprayed Khan with a fire extinguisher, others struck him with their fists and took his knife, and another, a Polish chef named ukasz, harried him with a five-foot narwhal tusk. [...]

Summary : On Friday afternoon, a man named Faisal Khan entered a Cambridge University building and started attacking people with a knife and a fire extinguisher .

Question 1: What did the attacker have?

Article answer: a large knife Summary answer: a knife and a fire extinguisher

Question 2: When did the attack take place?

Article answer: Friday Summary answer: Friday afternoon

Question 3: What is the attacker's name?

Article answer: Usman Khan Summary answer: Faisal Khan

Question 4: Where did the attack take place?

Article answer: Fishmongers' Hall Summary answer: Cambridge University building

Article: In findings published on Wednesday in the journal PLOS ONE, an international team of scientists report ancient Egyptians captured sacred ibises (Threskiornis aethiopicus) from the wild for use in ritual sacrifice rather than domesticating the birds. [...] The team collected DNA samples from mummified birds collected from six separate catacombs including sites at Abydos, Saqqara, and Tuna el-Gebel with permission from the Egyptian Ministry of State for Antiquity, and several museums offered to send tissue samples from the mummified ibises in their collections. [...]

Summary : Archaeologists have used DNA samples from ancient ibis birds to determine whether the birds were domesticated or sacrificed in ancient Egypt

Question 1: Archaeologists have used what to determine whether the birds were domesticated ?

Article Answer: hatchery structures Summary Answer: DNA samples

Question 2: Who used DNA samples to determine whether the birds were domesticated?

Article Answer: [NO ANSWER] Summary Answer: Archaeologists

Question 3: What are archeologists using to determine whether the birds were domesticated?

Article Answer: DNA samples Summary Answer: DNA samples

Question 4: Where were the birds found?

Article Answer: six separate catacombs Summary Answer: ancient Egypt

Table 6: Example questions and answers generated when computing QAGS. The questions are overwhelmingly fluent and relevant. The answers indicate which tokens in the summary are factually consistent or inconsistent. The news articles are originally from https://en.wikinews.org/wiki/Bystanders_foil_knife-weilding_ man_on_London_Bridge_with_fire_extinguisher,_whale_tusk and https://en.wikinews.org/ wiki/Ancient_Egyptians_collected_wild_ibis_birds_for_sacrifice,_says_study.

including fluency, readability, or factual recall. We therefore recommend using QAGS in conjunction with complementary evaluation metrics.

The choices of QG and QA models in QAGS are particular to abstractive summarization and may require adaptation to be used for other conditional text generation tasks. For example, we expect that extractive summarization models may obtain nearly perfect QAGS scores because facts and statements are directly copied from the source article.

\section{Related Work}

Automatic summarization and its evaluation are long-standing lines of work in NLP, dating at least as far back as the Document Understanding Conferences (Chali and Kolla, 2004). The primary evaluation metric then and now is ROUGE (Lin, 2004), though much work has demonstrated the limited ability of ROUGE and its relatives to evaluate summaries (Dorr et al., 2004; Liu and Liu, 2009; Kedzie et al., 2018, i.a.). Other metrics have focused on specific aspects of summarization quality, including content selection (Nenkova and Passonneau, 2004), relevance prediction (Daume III and Marcu, 2005), and many more.

The idea of evaluating summaries by their ability to answer a set of questions is also long-standing (Mani et al., 1999). Like our work, Eyal et al. 
(2019) and Scialom et al. (2019) extend this line of work by incorporating neural network modules. We diverge from these works in two important ways. First, both works use Cloze-style questions, which are generated by masking entities in either the source document or the reference summary. We instead generate the questions with a model, allowing a much greater range of questions. Second, we produce questions conditioned on the generated summary, rather than the reference summary or source article. Producing questions from the generated summary is more appropriate for verifying the accuracy of the text, whereas using the reference or source measures content selection.

There has been a recent resurgence of work leveraging NLU models for evaluating the factuality of generated text. Goodrich et al. (2019) use information extraction models to measure factual overlap, but facts are restricted to pre-defined schemas. Falke et al. (2019) investigate the use of NLI models to evaluate the factual correctness of CNN/DM summaries, and conclude that current NLI models are too brittle to be reliably used in this manner. Kryscinski et al. (2019b) train a NLI-based factchecking model by building a dataset of factual inconsistencies based on noise heuristics. Our QA approach allows a finer-grained analysis, because NLI operates on complete sentences, whereas QAGS can ask many different questions about the same sentence.

\section{Conclusion}

We introduce a framework for automatically detecting factual inconsistencies in conditionally generated texts and use this framework to develop QAGS, a metric for measuring inconsistencies in abstractive summarization. QAGS correlates with human judgments of factuality significantly better than standard automatic evaluation metrics for summarization, and outperforms related NLI-based approaches to factual consistency checking. QAGS is naturally interpretable: The questions and answers produced in computing QAGS indicate which tokens in a generated summary are inconsistent and why.

The framework we present is general, and extending it to other conditional text generation tasks such as image captioning or machine translation is a promising directions. Inspecting the generated questions and answers, we identify the transfer ability of QA models and the rigidity of F1 score as a measure of answer similarity as two key performance bottlenecks. We expect improvements in either would straightforwardly improve the quality of QAGS evaluation. Additionally, incorporating a content selection mechanism to focus the generated questions on salient facts is a promising direction. Overall, we believe QAGS demonstrates the potential of this framework to quantify and incentivize factually consistent text generation.

\section{Acknowledgments}

We thank Margaret Li and Jack Urbanek for help with Amazon Mechanical Turk. AW is supported by the National Science Foundation Graduate Research Fellowship Program under Grant No. DGE 1342536. Any opinions, findings, and conclusions or recommendations expressed in this material are those of the authors and do not necessarily reflect the views of the National Science Foundation. KC was partly supported by Samsung Advanced Institute of Technology (Next Generation Deep Learning: from pattern recognition to AI) and Samsung Research (Improving Deep Learning using Latent Structure).

\section{References}

Ekaterina Ageeva, Mikel L. Forcada, Francis M. Tyers, and Juan Antonio Pérez-Ortiz. 2015. Evaluating machine translation for assimilation via a gap-filling task. In Proceedings of the 18th Annual Conference of the European Association for Machine Translation, pages 137-144, Antalya, Turkey.

Ziqiang Cao, Furu Wei, Wenjie Li, and Sujian Li. 2018. Faithful to the original: Fact aware neural abstractive summarization. In Thirty-Second AAAI Conference on Artificial Intelligence.

Yllias Chali and Maheedhar Kolla. 2004. Summarization techniques at duc 2004. In In Proceedings of the Document Understanding Conference. Citeseer.

Qian Chen, Xiaodan Zhu, Zhen-Hua Ling, Si Wei, Hui Jiang, and Diana Inkpen. 2017. Enhanced lstm for natural language inference. In Proceedings of the 55th Annual Meeting of the Association for Computational Linguistics (Volume 1: Long Papers), pages 1657-1668.

Yen-Chun Chen and Mohit Bansal. 2018. Fast abstractive summarization with reinforce-selected sentence rewriting. In Proceedings of the 56th Annual Meeting of the Association for Computational Linguistics (Volume 1: Long Papers), pages 675-686.

Hal Daume III and Daniel Marcu. 2005. Bayesian summarization at duc and a suggestion for extrinsic 
evaluation. In Proceedings of the Document Understanding Conference, DUC-2005, Vancouver, USA.

Jacob Devlin, Ming-Wei Chang, Kenton Lee, and Kristina Toutanova. 2019. Bert: Pre-training of deep bidirectional transformers for language understanding. In Proceedings of the 2019 Conference of the North American Chapter of the Association for Computational Linguistics: Human Language Technologies, Volume 1 (Long and Short Papers), pages 4171-4186.

Bonnie Dorr, Christof Monz, Douglas Oard, David Zajic, and Richard Schwartz. 2004. Extrinsic evaluation of automatic metrics for summarization. Technical report, MARYLAND UNIV COLLEGE PARK INST FOR ADVANCED COMPUTER STUDIES.

Xinya Du, Junru Shao, and Claire Cardie. 2017. Learning to ask: Neural question generation for reading comprehension. In Proceedings of the 55th Annual Meeting of the Association for Computational Linguistics (Volume 1: Long Papers), pages 13421352.

Matan Eyal, Tal Baumel, and Michael Elhadad. 2019. Question answering as an automatic evaluation metric for news article summarization. In Proceedings of the 2019 Conference of the North American Chapter of the Association for Computational Linguistics: Human Language Technologies, Volume 1 (Long and Short Papers), pages 3938-3948.

Tobias Falke, Leonardo FR Ribeiro, Prasetya Ajie Utama, Ido Dagan, and Iryna Gurevych. 2019. Ranking generated summaries by correctness: An interesting but challenging application for natural language inference. In Proceedings of the 57th Conference of the Association for Computational Linguistics, pages 2214-2220.

Angela Fan, Mike Lewis, and Yann Dauphin. 2018. Hierarchical neural story generation. In Proceedings of the 56th Annual Meeting of the Association for Computational Linguistics (Volume 1: Long Papers), pages 889-898.

Sebastian Gehrmann, Yuntian Deng, and Alexander Rush. 2018. Bottom-up abstractive summarization. In Proceedings of the 2018 Conference on Empirical Methods in Natural Language Processing, pages 4098-4109.

Ben Goodrich, Vinay Rao, Peter J. Liu, and Mohammad Saleh. 2019. Assessing the factual accuracy of generated text. In Proceedings of the 25th ACM SIGKDD International Conference on Knowledge Discovery \& Data Mining, KDD '19, pages 166175, New York, NY, USA. ACM.

Karl Moritz Hermann, Tomas Kocisky, Edward Grefenstette, Lasse Espeholt, Will Kay, Mustafa Suleyman, and Phil Blunsom. 2015. Teaching machines to read and comprehend. In Advances in neural information processing systems, pages 1693-1701.
Ari Holtzman, Jan Buys, Maxwell Forbes, and Yejin Choi. 2019. The curious case of neural text degeneration. arXiv preprint arXiv:1904.09751.

Chris Kedzie, Kathleen McKeown, and Hal Daume III. 2018. Content selection in deep learning models of summarization. In Proceedings of the 2018 Conference on Empirical Methods in Natural Language Processing, pages 1818-1828.

Kalpesh Krishna and Mohit Iyyer. 2019. Generating question-answer hierarchies. In Proceedings of the 57th Annual Meeting of the Association for Computational Linguistics, pages 2321-2334, Florence, Italy. Association for Computational Linguistics.

Wojciech Kryscinski, Nitish Shirish Keskar, Bryan McCann, Caiming Xiong, and Richard Socher. 2019a. Neural text summarization: A critical evaluation. In Proceedings of the 2019 Conference on Empirical Methods in Natural Language Processing, Volume 1 (Long and Short Papers).

Wojciech Kryscinski, Bryan McCann, Caiming Xiong, and Richard Socher. 2019b. Evaluating the factual consistency of abstractive text summarization.

Alon Lavie and Abhaya Agarwal. 2007. Meteor: An automatic metric for mt evaluation with high levels of correlation with human judgments. In Proceedings of the Second Workshop on Statistical Machine Translation, pages 228-231. Association for Computational Linguistics.

Logan Lebanoff, John Muchovej, Franck Dernoncourt, Doo Soon Kim, Seokhwan Kim, Walter Chang, and Fei Liu. 2019. Analyzing sentence fusion in abstractive summarization. In Proceedings of the 2nd Workshop on New Frontiers in Summarization, pages 104-110.

Mike Lewis, Yinhan Liu, Naman Goyal, Marjan Ghazvininejad, Abdelrahman Mohamed, Omer Levy, Ves Stoyanov, and Luke Zettlemoyer. 2019. BART: Denoising sequence-to-sequence pretraining for natural language generation, translation, and comprehension. arXiv preprint 1910.13461.

Chin-Yew Lin. 2004. Rouge: A package for automatic evaluation of summaries. In Text summarization branches out, pages 74-81.

Chia-Wei Liu, Ryan Lowe, Iulian Serban, Mike Noseworthy, Laurent Charlin, and Joelle Pineau. 2016. How not to evaluate your dialogue system: An empirical study of unsupervised evaluation metrics for dialogue response generation. In Proceedings of the 2016 Conference on Empirical Methods in Natural Language Processing, pages 2122-2132.

Feifan Liu and Yang Liu. 2009. Exploring correlation between rouge and human evaluation on meeting summaries. IEEE Transactions on Audio, Speech, and Language Processing, 18(1):187-196. 
Ilya Loshchilov and Frank Hutter. 2018. Decoupled weight decay regularization.

Inderjeet Mani, David House, Gary Klein, Lynette Hirschman, Therese Firmin, and Beth M Sundheim. 1999. The tipster summac text summarization evaluation. In Ninth Conference of the European Chapter of the Association for Computational Linguistics.

Alexander Miller, Will Feng, Dhruv Batra, Antoine Bordes, Adam Fisch, Jiasen Lu, Devi Parikh, and Jason Weston. 2017. Parlai: A dialog research software platform. In Proceedings of the 2017 Conference on Empirical Methods in Natural Language Processing: System Demonstrations, pages 79-84.

Ramesh Nallapati, Bowen Zhou, Cicero dos Santos, Çağlar Gulçehre, and Bing Xiang. 2016. Abstractive text summarization using sequence-to-sequence RNNs and beyond. In Proceedings of The 20th SIGNLL Conference on Computational Natural Language Learning, pages 280-290, Berlin, Germany. Association for Computational Linguistics.

Shashi Narayan, Shay B. Cohen, and Mirella Lapata. 2018. Don't give me the details, just the summary! Topic-aware convolutional neural networks for extreme summarization. In Proceedings of the 2018 Conference on Empirical Methods in Natural Language Processing, Brussels, Belgium.

Ani Nenkova and Rebecca Passonneau. 2004. Evaluating content selection in summarization: The pyramid method. In Proceedings of the human language technology conference of the north american chapter of the association for computational linguistics: Hlt-naacl 2004, pages 145-152.

Myle Ott, Sergey Edunov, Alexei Baevski, Angela Fan, Sam Gross, Nathan Ng, David Grangier, and Michael Auli. 2019. Fairseq: A fast, extensible toolkit for sequence modeling. NAACL HLT 2019, page 48 .

Kishore Papineni, Salim Roukos, Todd Ward, and WeiJing Zhu. 2002. Bleu: a method for automatic evaluation of machine translation. In Proceedings of the 40th annual meeting on association for computational linguistics, pages 311-318. Association for Computational Linguistics.

Gabriel Pereyra, George Tucker, Jan Chorowski, Lukasz Kaiser, and Geoffrey Hinton. 2017. Regularizing neural networks by penalizing confident output distributions.

Pranav Rajpurkar, Robin Jia, and Percy Liang. 2018. Know what you dont know: Unanswerable questions for squad. In Proceedings of the 56th Annual Meeting of the Association for Computational Linguistics (Volume 2: Short Papers), pages 784-789.

Thomas Scialom, Sylvain Lamprier, Benjamin Piwowarski, and Jacopo Staiano. 2019. Answers unite! unsupervised metrics for reinforced summarization models. In Proceedings of the 2019 Conference on Empirical Methods in Natural Language Processing and the 9th International Joint Conference on Natural Language Processing (EMNLPIJCNLP), pages 3237-3247.

Kaitao Song, Xu Tan, Tao Qin, Jianfeng Lu, and TieYan Liu. 2019. Mass: Masked sequence to sequence pre-training for language generation. In International Conference on Machine Learning, pages 5926-5936.

Adam Trischler, Tong Wang, Xingdi Yuan, Justin Harris, Alessandro Sordoni, Philip Bachman, and Kaheer Suleman. 2017. Newsqa: A machine comprehension dataset. In Proceedings of the 2nd Workshop on Representation Learning for NLP, pages 191-200.

Sean Welleck, Jason Weston, Arthur Szlam, and Kyunghyun Cho. 2019. Dialogue natural language inference. In Proceedings of the 57th Annual Meeting of the Association for Computational Linguistics, pages 3731-3741, Florence, Italy. Association for Computational Linguistics.

Adina Williams, Nikita Nangia, and Samuel Bowman. 2018. A broad-coverage challenge corpus for sentence understanding through inference. In Proceedings of the 2018 Conference of the North American Chapter of the Association for Computational Linguistics: Human Language Technologies, Volume 1 (Long Papers), pages 1112-1122.

Thomas Wolf, Lysandre Debut, Victor Sanh, Julien Chaumond, Clement Delangue, Anthony Moi, Pierric Cistac, Tim Rault, Rémi Louf, Morgan Funtowicz, et al. 2019. Transformers: State-of-the-art natural language processing. arXiv preprint 1910.03771.

Tianyi Zhang, Varsha Kishore, Felix Wu, Kilian Q Weinberger, and Yoav Artzi. 2019. Bertscore: Evaluating text generation with bert. arXiv preprint 1904.09675. 


\section{A Human Evaluation Task Design}

We restrict our pool of workers to US-based workers. Workeres are required to have at least 1000 approved HITs with an acceptance rate of at least $98 \%$.

The base reward for our task is $\$ 0.15$. For each summary, we include automatic quality checks including

- Time checks: workers who complete the task under 30s fail the check

- Attention checks: we include exact copies of article sentences and corrupted mixtures of two article sentences as positive and negative control task. If a worker fails to answer both of these examples correctly, they fail the check

- Explanation checks: For each sentence in the summary, the worker is required to provide a short explanation of their decision

If a worker passes all checks, they are awarded a $\$ 0.85$ bonus, totalling $\$ 1.00$ per correct annotation. According to turkerview.com, workers of our HIT are paid well in excess of $\$ 15.00$ on average.

We show our annotation interfaces for the annotation task for CNN/DM and XSUM respectively in Figures 2 and 3. We use slightly different instructions to accommodate for the quirks of each dataset. For XSUM, we prepend the reference "summary" back onto the source article, as without it, workers were struggling to identify factual inconsistencies.

\section{B Model and Generation Details}

Question Generation We fine-tune BART for question generation using the same tuning hyperparameters as the original work. We optimize label smoothed cross entropy with smoothing parameter 0.1 (Pereyra et al., 2017) and a peak learning rate of $2 \mathrm{e}-5$. We optimize for 100k steps with 5k warmup steps, and use the model with the best perplexity on the development set.

To turn NewsQA into an answer conditional QG dataset, we concatenate the answer to the source article with a special marker token in between. We then concatenate another special marker token and the question. At test time, we get 10 named entities and noun phrases as answer candidates using the en-web-sm spaCy model. We randomly sample 10 if there are more than 10, and randomly duplicate some answers if there are fewer than 10 . The model predicts the question after seeing an answer and the article.

During decoding, we use beam search with beam size 10 , length penalty 1.0 , and trigram repetition blocking. Generations have minimum length 8 and max length 60 .

To filter the questions, we first use simple heuristics, including removing

- everything after the first question mark in a question

- exact duplicates

- questions shorter than three tokens long

For the remaining questions, we use our QA model to answer each question and we remove questions for which the QA model deems unanswerable. We then take the top 20 most probable questions, random sampling some of the filtered questions if there were too few.

Question Answering We fine-tune BERT for question answering following the original work. Similar to the QG setting, we append the question and answer to the source article with intervening special marker tokens. We optimize using AdamW (Loshchilov and Hutter, 2018) with initial learning rate $5 \mathrm{e}-5$. We train for 3 epochs, with a warmup ratio of 0.1 . We use the model with the best development set performance. 


\section{Is the sentence supported by the article? \\ You are currently at comparison $1 / 5$ In this task, you will read an article on the left and a series of sentences on the right. \\ The task is to determine if the sentences are factually correct given the contents of the anticle. Many \\ sentences corthe article-Be careful as soxt copied directly \\ be combinations of two different parts of the article, \\ resulting in sentences that overall aren't supported by the article. Some article sentences may seem

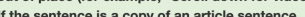 including one of hicse ser treat it as factually supported. Othervise, if the sentence doesn't make sense, you should mark as not supported. Also note that the article may be cut off at the end \\ If you successtully complete all tasks, we will awarc}

Is the sentence factually supported by the article ?

Yes

Please provide a justification for your choice (a few words or a sentence).
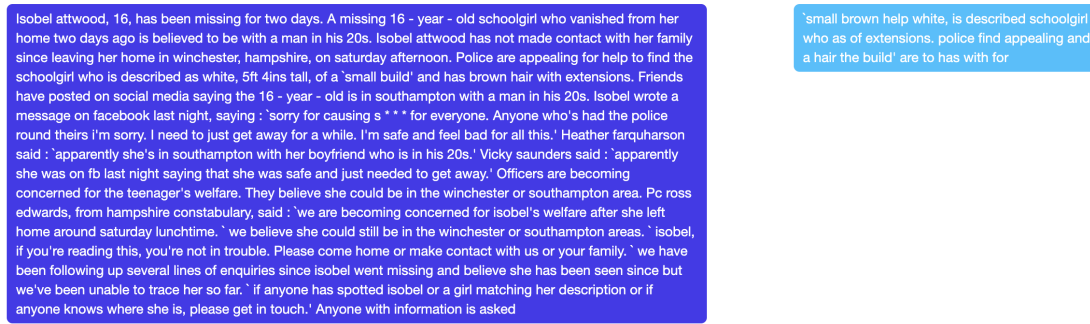

Please enter here.

Figure 2: Annotation interface and instructions for CNN/DM factual consistency task.

Is the sentence supported by the article?

You are currently at comparison $1 / 3$

In this task, you will read an article on the left and a

series of sentences on the right.

The task is to determine if the sentences are factually correct given the contents of the article. All parts

article to be considered correct. For example inc

sentence discusses "John Smith" but the article only

talks about "Mr. Smith", the fact that the person's first

name is John is NOT supported. Or, if the senten

mentions a 15-year-old girl but the article only

discusses a young gin, the fact that she is 15 is NO

combining facts from many different parts of the

article, so read the entire article closely. If the

sentence directly copies the article, you should mark it

as supported. If the sentence doesn't make sense, you

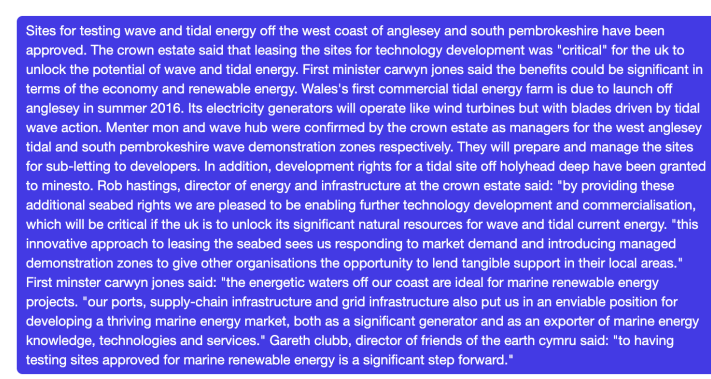

Is the sentence factually supported by the article ?

Yes

Pease provide a justification for your choice (a few words or a sentence).

Please enter here.

Figure 3: Annotation interface and instructions for XSUM factual consistency task. 